\title{
Looking at object-substitution masking in depth and motion: Toward a two-object theory of object substitution
}

\author{
TODD A. KAHAN and ANDREA S. LICHTMAN \\ Bates College, Lewiston, Maine
}

\begin{abstract}
When attention is divided, a briefly presented target surrounded by four small dots is difficult to identify when the dots persist beyond target offset, but not when these dots terminate with the target. This object-substitution masking effect likely reflects processes at both the image level and the object level. At the image level, visual contours of the mask make feature extraction difficult. Recent data (Lleras \& Moore, 2003) suggest that, at the object level, an object file is created for the target-plus-mask, and this single-object token later morphs into a single-object token containing the mask alone. In the present experiments, we used stimuli presented in 3-D space and apparent motion; the results indicate that object-substitution masking also arises when the mask and the target are represented in two separate object tokens and the mask token interferes with the target token.
\end{abstract}

Many visual masking effects (e.g., masking by pattern, illumination, and metacontrast) occur at the image level and reflect low-level processes that arise during stimulus encoding and feature extraction (Breitmeyer, 1984). Another form of masking has been identified that may also reflect object-level interference (Di Lollo, Enns, \& Rensink, 2000; Enns \& Di Lollo, 1997, 2000; but see also Breitmeyer \& Ogmen, 2000). In the standard object-level masking paradigm, participants are asked to identify a briefly presented target object that occurs in a randomly selected location and is surrounded by four spatially adjacent dots arranged in a discernible square. If the dots terminate with the target, accuracy is high; if the dots have a delayed offset, accuracy is poor. This effect, referred to as object-substitution masking, might reflect both imagelevel and object-level interference.

At the image level, visual contours of the mask may disrupt target identification (Breitmeyer \& Ogmen, 2000). At the object level, the target and the mask may interact through reentrant pathways in the visual cortex. According to Di Lollo et al. (2000), when the target and the dots are presented, a perceptual hypothesis regarding the target's shape begins to form, and this percept is regularly updated. Higher level brain regions (where the percept is being formed) scan lower level regions for additional information. This top-down check is theorized to occur in an iterative manner, whereby higher level brain regions are cyclically updated with information received from lower level regions. If the dots are removed upon target

The authors thank Vince Di Lollo, Jim Enns, Kathy Mathis, Cathleen Moore, and Jim Neely for their helpful comments. Correspondence should be addressed to T. A. Kahan, Department of Psychology, Bates College, Lewiston, ME 04240 (e-mail: tkahan@bates.edu). offset, no additional information is added to the perceptual hypothesis, and the intact, albeit uncertain, percept is entered into consciousness. If the dots have a delayed offset, cyclical updates will result in the replacement of the target object with the masking object (i.e., masking by object substitution).

Because this delayed-offset masking effect occurs with a relatively sparse mask when the contours of the mask and the target are too distant for effective image-level masking, and because the effect is maximal under conditions of divided attention, Di Lollo et al. (2000) argue that it mostly reflects object-level processes. As such, this masking effect may provide clues regarding the way objects are updated and the way objects interact in the mind.

Using apparent motion displays, Lleras and Moore (2003) helped clarify the nature of object-substitution masking. Across several experiments, masking was obtained when the dot mask was located adjacent to, rather than surrounding, the target, as long as the spatiotemporal properties supported the percept of the target moving toward the mask. According to the authors, one object file (or token) is created for the target, and the contents of this file are updated with the mask (for a review of "object files," see Kahneman, Treisman, \& Gibbs, 1992). Thus, in Lleras and Moore's account, object-level masking does not reflect the interplay of two separate object tokens; rather it reflects the updating of a single object token, which we hereafter refer to as single-object morphing. If apparent motion was not perceived and the mask was seen as a distinct object, no masking was obtained. Hence, masking was obtained only when the target object was seen morphing into, or becoming, the mask (one object that changes its form over time).

Lleras and Moore's (2003) data clearly demonstrate that when perceived motion occurs, object-level masking can 
arise from single-object morphing. Hence, it is possible that object-level masking effects are solely the consequence of single-object morphing. However, because the trailing dot mask did not surround the target in Lleras and Moore's experiments, their data do not preclude that when the standard static procedure is used, object-level masking can occur when separate object tokens are created for the target and the mask, and the mask's object token interferes with the target's object token. It is important to note two things. First, the two-object interference account that we directly tested in the present investigation does not deny that masking can also arise from single-object morphing. ${ }^{1}$ Second, in fairness, Lleras and Moore explicitly and successfully designed their experiments to test whether masking from single-object morphing can occur and not to test whether masking from two-object interference can also occur.

\section{EXPERIMENT 1}

If the mask forms a unique token, can masking be found? To answer this question, in Experiment 1, we had the participants view static stereoscopic displays in which the mask and the target were each, independently, viewed as being near or far, and we manipulated the offset delay of the mask. Stereoscopic displays were chosen to increase the probability that the participants would perceive and represent objects located in different depth planes as unique tokens. If the object-level component of object substitution masking is produced solely by single-object morphing, and if the target is more often seen as becoming the mask when the two are located at the same depth of view, masking should be maximal when the target and the mask are perceived in the same depth plane. The target should be seen as moving into (or becoming) the mask more often when the target and the mask are located in the same depth plane, because, in this situation, the target and the mask will more likely be seen as occupying the same object file. Congruent with this claim, $\mathrm{He}$ and $\mathrm{Na}-$ kayama (1994) found that the perception of apparent motion is more likely when stimuli are located on the same surface. In their experiments, although participants did perceive motion across depth planes (e.g., when a background slanting surface was presented on which the objects could appear to move), participants had a propensity for seeing motion within the same plane rather than across depth planes when no surface was provided and the disparity between depth planes was large. In the present experiment, when the target and the mask were presented in different depth planes, the disparity was obvious, and no slanting surfaces were presented. Under these conditions, perception of motion across depth should be unlikely, the target and the mask should be seen as separate object tokens, and masking should not be found. In contrast, if the object-level component of object-substitution masking is produced in part by interference between two separate object files, whereby the object file for the mask interferes with the object file for the target, then masking ought to occur when the target and the mask are perceived in the same depth plane (near or far) and when the mask is perceived as being closer than the target. When the target is perceived in front of the mask, very little masking ought to occur because the continued presence of the masking dots will not occlude the target's mental representation. (The specific mechanism that might be responsible for two-object interference will be explained in the General Discussion section.) Thus, the two-object interference account predicts masking across depth planes, whereas the single-object morphing account does not; these predictions were tested.

\section{Method}

Participants. Twenty-one students from Bates College participated for extra credit in an introductory psychology course. All reported having normal or corrected-to-normal vision.

Materials and Procedure. The participants viewed stimuli through a Brewster stereoscope affixed to a computer monitor set at $1,024 \times 768$ pixel resolution. The participant's eyes were located $14 \mathrm{~cm}$ from the screen, and the stereoscope contained +5.0 diopter lenses, which helped fuse the images and magnified the screen $125 \%$. The presentation of stimuli and the recording of responses were controlled using E-Prime software v1.1 (Schneider, Eschman, \& Zuccolotto, 2002a, 2002b) running on a Dell Pentium IV 2 GHz PC.

Each of the four frames in the experiment was composed of two images, each of which was exclusively presented to one eye (see Figure 1). In the first fixation frame, a " $+"\left(9 \times 9\right.$ pixels; $\left.1.3^{\circ}\right)$ was displayed for $500 \mathrm{msec}$. This was immediately followed, in Frame 2, by four diamonds $\left(39 \times 39\right.$ pixels; $\left.5.6^{\circ}\right)$, each of which was centered on a corner of an imaginary square $\left(122 \times 122\right.$ pixels; $\left.17.4^{\circ}\right)$. One of these, the target, was missing either the left or the right corner ( 7 pixels were cut from the missing corner; $1^{\circ}$ ) and appeared floating either nearer or farther away than the remaining three diamonds, which appeared at the horopter. The target was always surrounded by four dots (10 pixels in diameter; $\left.1.4^{\circ}\right)$ that were arranged in a square configuration $\left(69 \times 69\right.$ pixels; $\left.9.9^{\circ}\right)$. The nearest distance between the dot mask and the target was $3.1^{\circ}$. To produce the perception of depth, the mask and target were independently shifted outward or inward by 5 pixels $\left(0.7^{\circ}\right)$. This created three depth planes. The full diamonds were always perceived in the middle depth plane, whereas the target and the mask were independently perceived in either the nearest or the farthest depth plane. The target was (1) missing either the left or the right corner, (2) located in any one of the four quadrants, (3) surrounded by a dot mask that was perceived as being either near or far, and (4) itself perceived as being either near or far; this created 32 combinations. Frame 2 was presented for $16 \mathrm{msec}$. Following the target frame, the dot mask remained visible, in Frame 3, for 0, 80, 160, or $640 \mathrm{msec}$. In Frame 4, a blank screen was presented until the participants pressed either the "Q" or the "P" key to indicate whether the target was missing the left or the right corner, respectively. The participants responded to 384 randomly selected trials; each of the 32 combinations was presented 12 times. Self-paced breaks were provided after every 96 trials.

Preceding these 384 experimental trials, the participants completed 24 trials designed to measure perceived target-mask distance. During this block of trials, the participants were shown the fixation "+," described above, followed by a frame with four diamonds (one of which was the target) and the masking pattern. In this block, the target and the mask always appeared in the upper-left quadrant, and the participants were instructed to enter the distance they perceived between the mask and the target. Rather than displaying this image for $16 \mathrm{msec}$, as was done in the experiment proper, the figure remained on the screen until a response was entered. The participants responded by entering a distance, in centimeters; a ruler was pro- 


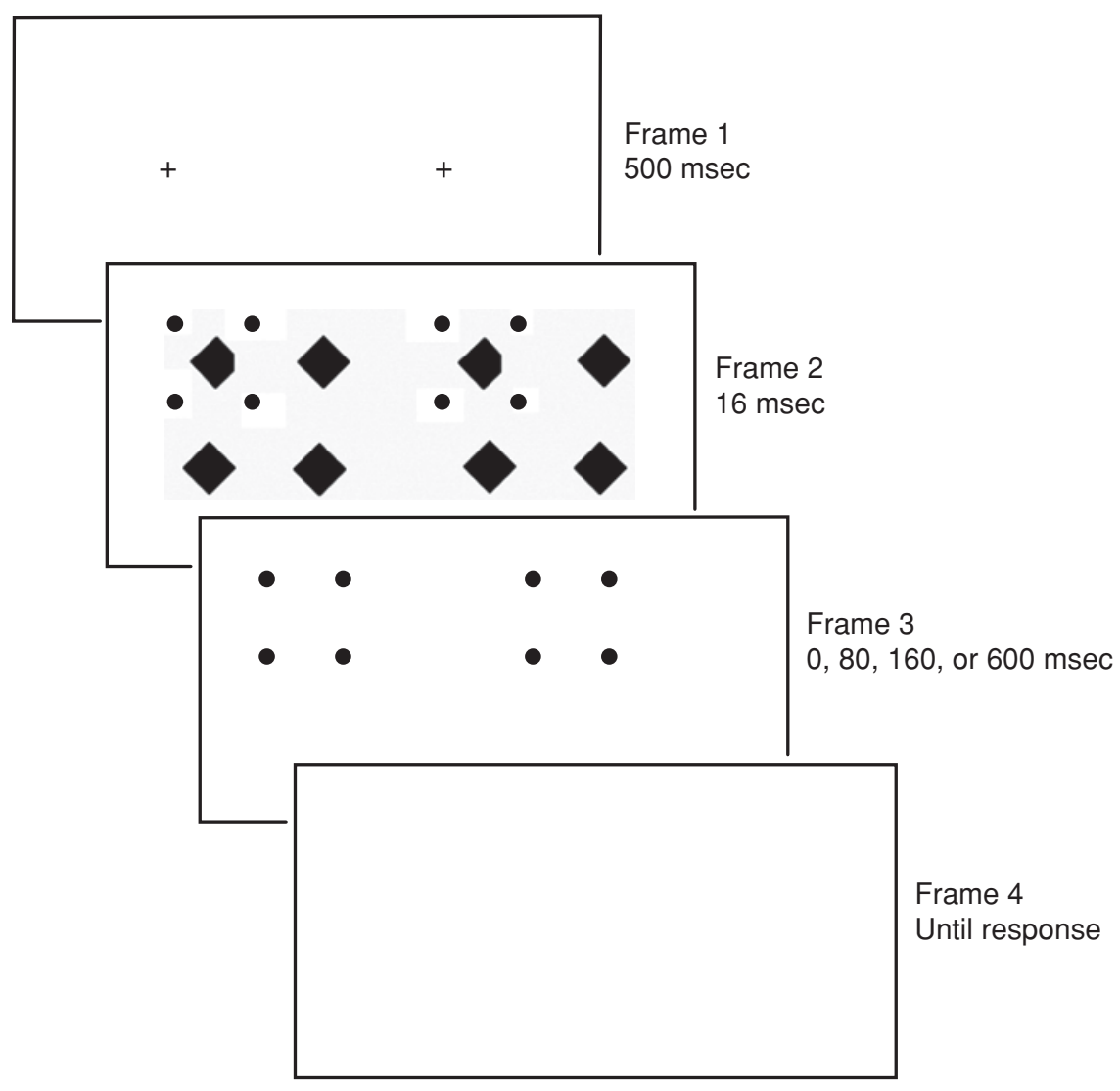

Figure 1. Sequence of events and timing parameters used in Experiment 1. In the trial depicted, the mask and the target are both perceived as being nearer than the full diamond shapes when viewed through a stereoscope.

vided as a reference. Eight of the 32 combinations were used (i.e., only figures with a target in the upper-left quadrant). Each of these figures was randomly presented three times.

\section{Results and Discussion}

Perceived target-mask distance. For the perceived target-mask distance data, the participants perceived the mask and the target as nearer when presented in the same depth plane $(M=0.79 \mathrm{~cm})$ relative to when presented in different depth planes $(M=7.1 \mathrm{~cm})[t(20)=6.7, p<$ $.0001]$. There were no differences in perceived distances between the two same-depth-plane conditions $(p=.93)$ or the two different-depth-plane conditions $(p=.53)$. Every participant $(N=21)$ perceived the average targetmask distance as being less in the same-depth conditions than in the different-depth conditions; this was significant by a sign test ( $p=.0000005)$.

Target accuracy. Four a priori ANOVAs examining masking effects in each depth condition were computed, with the alpha level set at .0125 (.05/4) to control for experimentwise error. Target accuracy, as a function of mask and target location, is presented in Figure 2. When both the target and the mask were perceived in the closest depth plane (shaded circles in Figure 2), performance decreased as mask duration increased $[F(3,60)=5.3, p=.003]$. Likewise, when both the target and the mask were perceived in the farthest depth plane (unshaded triangles in Figure 2), performance decreased with increases in mask duration $[F(3,60)=4.5, p=.006]$. These data are consistent with predictions from both single-object morphing and two-object interference theories of masking. However, as predicted by two-object interference accounts, but not the single-object morphing account, when the target and the mask were perceived in different depth planes, masking was obtained when the mask was perceived in front of the target (unshaded circles in Figure 2) $[F(3,60)=$ $4.3, p=.008]$. No masking was found when the target was perceived in front of the mask (shaded triangles in Figure 2) $[F(3,60)=0.85, p=.47]$. Thus, masking was found when the target and the mask were presented in different depth planes only if the mask was seen in front of the target, not when the mask was seen behind the target; the contrast for this interaction was significant $[F(1,20)=$ $4.63, p<.05]$.

It is clear that depth was perceived under these viewing conditions because the depth manipulation effectively eliminated object-substitution masking only when the target was perceived in front of the mask. Because masking 


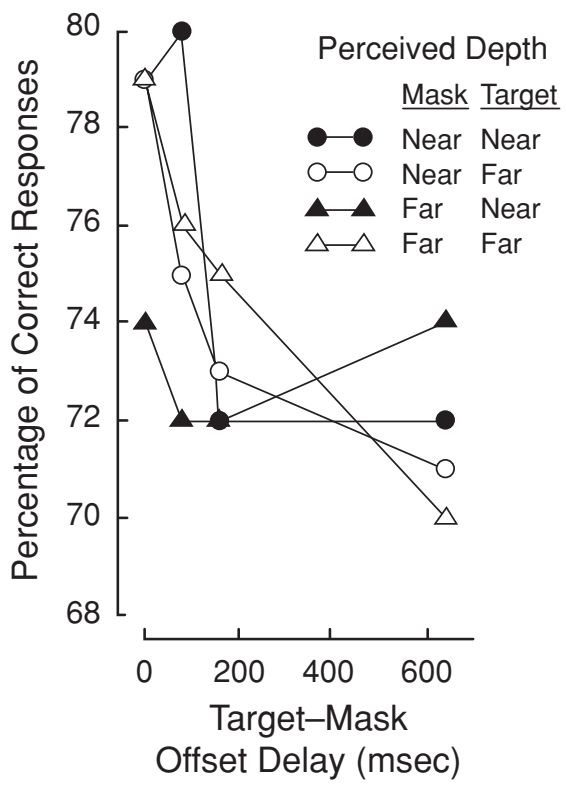

Figure 2. Experiment 1 accuracy rates as a function of mask duration and perceived depth of the mask and the target.

was obtained when the mask was perceived in front of the target, it cannot be the case that the participants were simply unable to resolve the displays (seeing overlapping shapes) when the target and the mask were presented in different depth planes; if this had been the case, no masking would have been found when the mask was seen in front of the target. However, because performance was numerically (though not statistically) lower in the immediate offset condition $(M=.74)$ when the target was seen in front of the mask, one might argue that our elimination of masking in this condition rests on a single data point. Yet it was not just one data point that differed when the target was seen in front of the mask (see shaded triangles in Figure 2); accuracy remained lower than the other depth conditions at the 80-msec offset delay and stayed at this level throughout, having the highest accuracy at the $640-\mathrm{msec}$ offset delay. Because the pattern of results across the different target-mask offset delays is fundamentally different when the target is seen in front of the mask compared with the other three conditions, and because this is the one condition for which masking was predicted to be eliminated, these results do not appear to reflect some chance peculiarity. Instead, the overall pattern of data appears to favor the two-object interference account over an account that says that masking is solely due to single-object morphing for static displays such as those used here.

Alternatively, if the targets were seen as moving into and becoming the masks, even in the different depth plane conditions (which were designed to prevent this from occurring), greater masking may have been obtained when the mask was seen in front of rather than behind the target, because the former rather than the latter would give rise to looming motion. Franconeri and Simons (2003) have dem- onstrated that looming objects capture attention whereas receding objects do not, and suggest that looming objects are more likely to be considered behaviorally urgent. It is well documented that object substitution masking increases as attention is directed toward the mask and away from the target (Neill, Hutchison, \& Graves, 2002). Therefore, if the target appeared to morph into the mask when the target and the mask were presented in different depth planes, attention might have been more strongly captured (and as a result object-substitution masking might have been larger) when looming motion relative to receding motion was perceived. As such, the data from our Experiment 1 do not, by themselves, falsify the solely singleobject morphing account. Because of this, we decided to conduct an experiment using a different methodology to ascertain better the contribution single-object morphing and two-object interference make to object-substitution masking. In Experiment 2, we used 2-D displays to eliminate the possibility of the participants' perceiving looming or receding motion.

\section{EXPERIMENT 2}

In Experiment 2, we sought further evidence regarding two-object interference and single-object morphing accounts of object-level masking, using a different approach originated by Lleras and Moore (2003), in which the mask appears to move. However, unlike in Lleras and Moore's experiments, in which the target and the mask started at the same spatial location and moved toward a new location occupied by the mask alone (supporting the perception of one object that changes over time), the present experiment also contained conditions in which the mask and the target started in separate locations and the masking object moved toward the position where the target object had been (supporting the perception of two objects that interfere with one another). Moore and Lleras (2005) used a similar method to create the perception of two objects; however, in their experiment, the masking dots moved past the location where the target had been, leaving the target location unmasked. In the present experiment, the mask did not move past the target location but instead moved to a position that surrounded the location where the target had been. In addition to this change, a different control technique was used. In prior research, two types of controls have been used to assess object-substitution masking (see Kahan \& Mathis, 2002). In the briefly masked control method, the mask always surrounds the target, and the mask's duration is manipulated. The briefly masked control method was used in the present Experiments 1 and 4; the target in the control condition was masked briefly (simultaneous offset) relative to the experimental conditions (delayed offset). In the unmasked control method, the mask either appeared in the same quadrant as the target or appeared in a different quadrant, and the mask persisted beyond target offset for a fixed duration. This control method was originated by Enns and Di Lollo (1997). The unmasked control method was used in the present Experiments 2 and 3; 
here, the target in the control condition was not masked. In addition to this change, stimuli were presented in 2-D rather than in depth (eliminating the possibility that the participants would perceive looming or receding motion, as could have occurred in Experiment 1), and the target was changed from a diamond missing either the left or the right corner to uppercase letters $\mathrm{E}$ or $\mathrm{F}$ (these targets were used by Neill et al., 2002). By having the mask either start with the target and move away (as one object would behave) or start away from the target and move toward it (as two objects would behave), we were able to examine the relative contributions that single-object morphing and two-object interference make to object-level masking. However, because image level effects will likely be greater when the target and the mask start in the same spatial location (their features will be closer to one another), ${ }^{2}$ the contribution of single-object morphing may be slightly overestimated when the mask starts with the target and moves away.

\section{Method}

Participants. Twenty students from Bates College participated for extra credit in an introductory psychology course. All reported having normal or corrected-to-normal vision.

Materials and Procedure. Experiments 2, 3, and 4 were conducted via the Internet, making it much easier to recruit participants by way of an e-mail announcement. These experiments were programmed using Macromedia Authorware v6.0. Authorware software has been used successfully to replicate experimental lab-based findings using Web delivery (McGraw, Tew, \& Williams, 2000; for a review of issues surrounding Web-based approaches to data collection, see Kraut et al., 2004). It should be noted that the timing of screen displays using Authorware software might not be as precise as that using E-Prime software. To eliminate any problems in interpreting the data that this might cause, we presented all of the conditions in a random order using a completely repeated measures design. To the extent that timing fluctuations occur, this variability will affect all of the conditions uniformly. Also, because the display resolution likely differed across participants, and because we could not control the distance between the participants' eyes and the computer monitor, display sizes are reported in pixels rather than in degrees of visual angle. Experiments 2, 3, and 4 all used Web delivery and fully randomized repeated measures designs. It was emphasized to the participants that it was important to complete the experiment in a quiet environment, free of distraction. This could range from dormitories to small on-campus computer laboratories. The weakness with this methodology is that variability might be added to the data in each experimental condition, making it less likely that any effects will be found. Importantly, every participant received all of the conditions in a completely randomized manner.

Refer to Figures 3 and 4 for the arrangement of stimuli and the sequence of events, respectively. A fixation point, "+" $(9 \times 9$ pixels), was displayed in the center of the screen and remained visible throughout the experiment. At the start of each trial, a masking pattern composed of four dots (each 11 pixels in diameter) and fitting within a discernible rectangle (51 pixels in width and 45 pixels in height) appeared in one of four quadrants. This masking pattern began at one of two distances from fixation and later moved to a new location. Two concentric rectangles defined these distances. In the outer positions, the mask was presented in a corner of an imaginary rectangle measuring 321 pixels in width and 269 pixels in height; in the inner positions, the imaginary rectangle measured 215 pixels in width and 179 pixels in height (see Figure 3; the open circle placeholders depicted in this figure were not displayed to the participants). This masking pattern was presented along with target

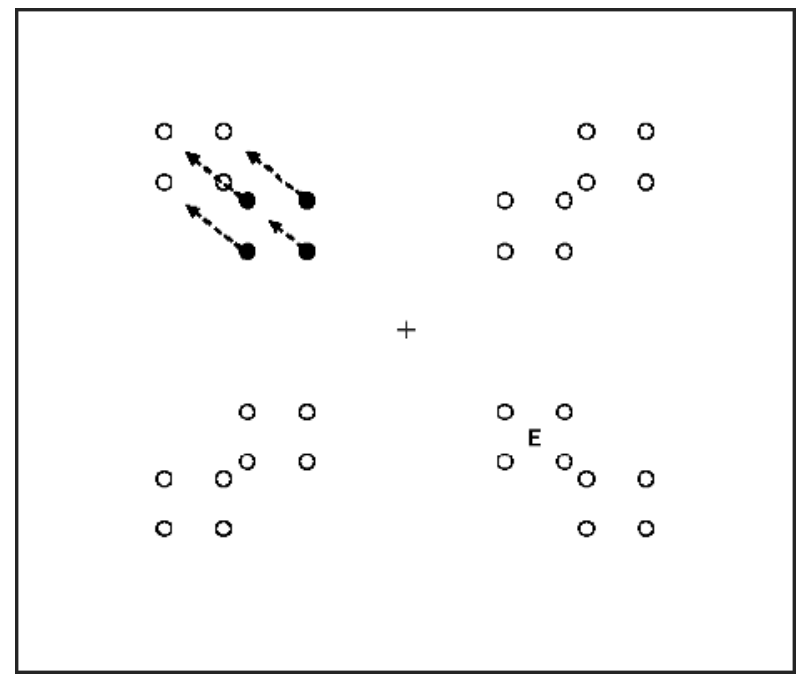

Figure 3. Arrangement of stimuli used in Experiments 2 and 3. Unfilled circle placeholders and dotted arrows were not displayed to the participants. The trial depicted is an unmasked away condition; the target letter $E$ and the masking dots are in different quadrants (unmasked), and the masking dots are moving outward (away from the target locations).

letter $\mathrm{E}$ or $\mathrm{F}$ (7 pixels in width and 10 pixels in height). The target was displayed randomly in one of four quadrants and appeared within the inner rectangle described above. In the masked condition (i.e., the mask and the target were presented in the same quadrant), the target letter was surrounded by the mask when the mask started at the inner location and was adjacent to the mask when the mask started at the outer location. In the unmasked condition, the mask and the target were presented in different quadrants. The target letter and the masking dots appeared simultaneously for $30 \mathrm{msec}$. Upon target offset, the dot mask moved from either the outer position to the inner position (referred to as toward movement, because the mask moved toward the target locations) or the inner position to the outer position (referred to as away movement, because the mask moved away from the target locations). Figure 4 depicts an unmasked away trial; the mask was located in a different quadrant (unmasked) and moved from the inner position to the outer position (away). The dot mask always moved within the same quadrant, and it took $500 \mathrm{msec}$ to traverse the distance; removing pixels from the trailing edge of the mask and adding them to the leading edge allowed the mask to traverse the distance in a smooth manner. When the masking pattern came to a halt, the participants were able to enter their target response using the "E" and "F" keys on the computer keyboard. Once a response was entered, the mask disappeared, and $500 \mathrm{msec}$ elapsed before the next trial began. The participants responded to 240 trials; the locations of the target and the mask were selected randomly on each trial. The target appeared in each of the four quadrants approximately 60 times, and the mask started from each of the eight possible positions (depicted in Figure 3) roughly 30 times. Self-paced breaks were given after every 60 experimental trials. Preceding the 240 experimental trials, the participants completed 10 practice trials that were also selected randomly. Data from the practice were discarded.

\section{Results and Discussion}

The percentages of correct target responses are depicted in the left portion of Figure 5 (see the slow-continuous motion conditions; these trials are referred to as slow be- 


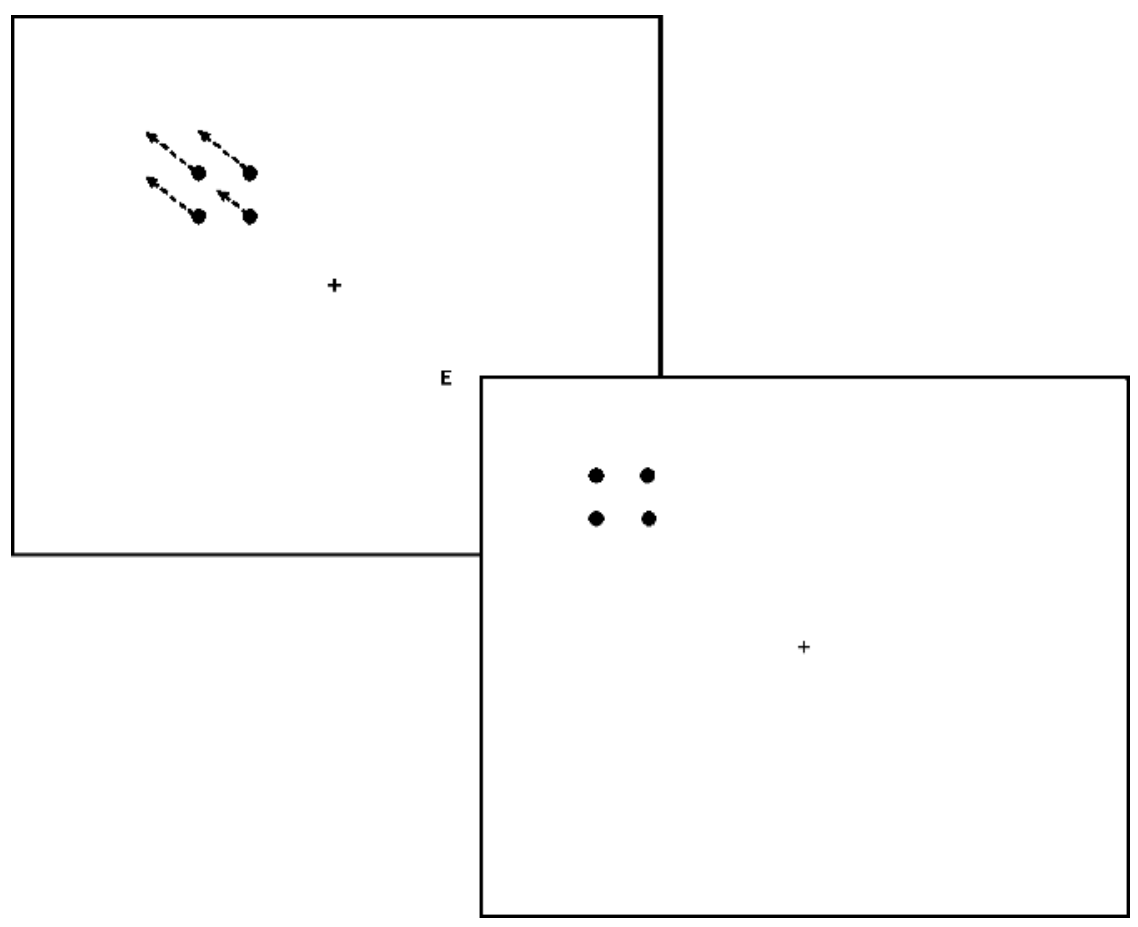

Figure 4. Sequence of events used in Experiments 2 and 3. Dotted arrows indicate the direction of mask motion and were not displayed to the participants. The target and the mask were presented together for $30 \mathrm{msec}$, and the mask began motion after target offset. In Experiment 2, the mask remained on the screen and traversed the distance in $500 \mathrm{msec}$ (slow-continuous motion). In Experiment 3, the mask disappeared with the target and reappeared at its final destination 30 msec later (fast-interrupted motion). The trial depicted here, analogous to the trial depicted in Figure 3, used an unmasked away condition.

cause motion was much more rapid in Experiment 3right portion of Figure 5-and continuous because the mask remained on the screen uninterrupted). A 2 (masking: masked vs. unmasked) $\times 2$ (direction of motion: toward vs. away) repeated measures ANOVA showed a main effect of masking $[F(1,19)=19.57, p<.001]$ but not of mask direction $[F(1,19)=.21, p>.05]$. Importantly, the masking effect was qualified by an interaction between masking and mask direction $[F(1,19)=8.89, p<.01]$. With a Fisher's LSD of $4.6 \%$ for this interaction, masking was significant when the dot mask moved toward the target locations (masking effect $=9 \%$ ) but not when it moved outward (masking effect $=0 \%$ ). Although we did not replicate Lleras and Moore's (2003) results in the away condition (possible reasons for this will be addressed in Experiments 3 and 4), these data provided converging evidence that object-level masking partly reflects two-object interference, using a different methodology.

Objects occupying separate spatial locations are more easily perceived as distinct than are those occupying the same location. When the mask and the target originated in separate locations, more masking was observed than when the mask initially surrounded the target. This effect (greater masking for the toward condition than for the away condition) does not reflect image-level variables because the mask and the target were closer when the mask initially surrounded the target (i.e., the away condition; see Note 2). Though these data support two-object interference, we were unable to replicate the single-object morphing effect reported by Lleras and Moore (2003), perhaps because motion was continuous, rather than interrupted. Because the masking pattern remained on the screen uninterrupted (whereas the target did not), it is possible that the target and the mask were always perceived as distinct objects because they did not have the same fate. To test this possibility, in Experiment 3, we used a mask that rapidly blinked with the target in one location and then appeared by itself in another location; the timing parameters were analogous to those used by Lleras and Moore. These timing parameters resulted in a mask that moved much more rapidly than that in Experiment 2.

\section{EXPERIMENT 3}

In Experiment 2, the mask remained on the screen uninterrupted, whereas in Lleras and Moore's (2003) experiments, the mask started in one location and, following a brief delay, reappeared in a new location. It is possible that a blank interval between presentation of the target-plusmask display and the mask-alone display is important in creating the perception that the target and the mask are part of the same object file. If the mask does not have the 


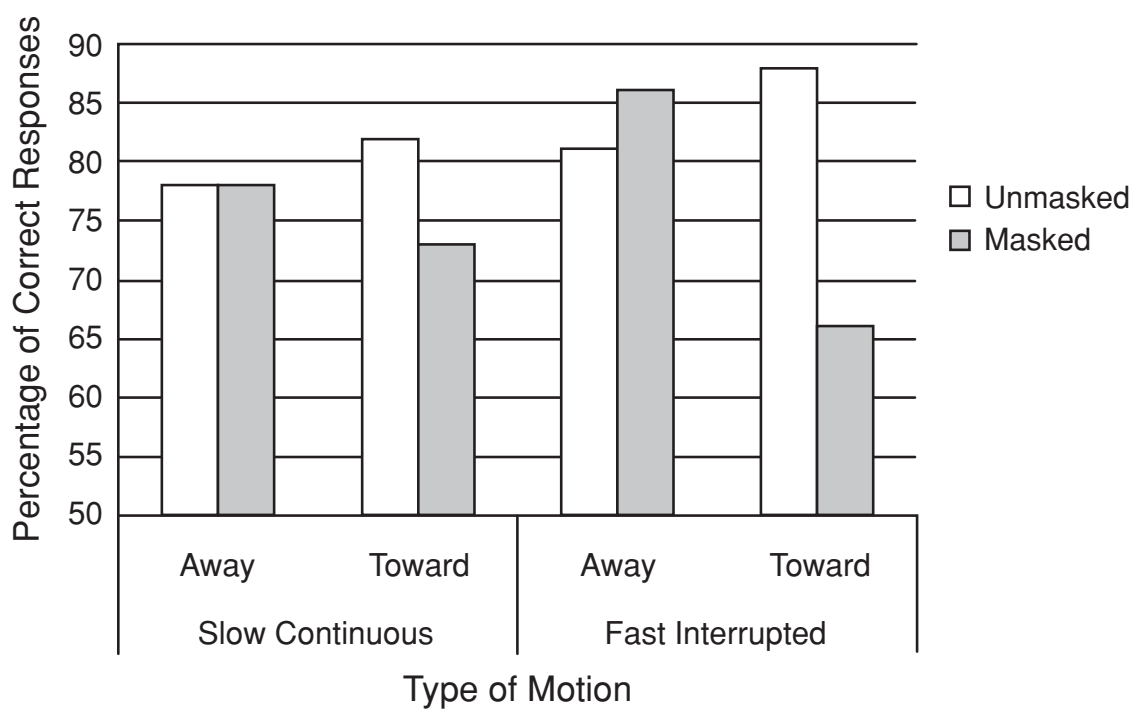

Figure 5. Accuracy rates for slow-continuous (Experiment 2) and fast-interrupted (Experiment 3) motion conditions as a function of the direction of the mask's motion (away or toward) and masking condition (unmasked or masked).

same fate as the target (i.e., does not disappear with the target), then the target might be coded as a unique object. Therefore, if a blank interval between the target-plus-mask display and the mask-alone display is needed for singleobject morphing to occur, using a mask that rapidly blinks in one location and then another (fast-interrupted motion) should allow us to uncover masking in the away motion condition. If two-object interference makes a unique contribution to object-substitution masking, we should continue to see masking in the toward motion condition.

\section{Method}

Participants. Nine students from Bates College participated for extra credit in an introductory psychology course. All reported having normal or corrected-to-normal vision.

Materials and Procedure. The materials and procedure were identical to those of Experiment 2 with one exception. Rather than the mask remaining on the screen and taking $500 \mathrm{msec}$ to travel to its final destination, the mask disappeared for $30 \mathrm{msec}$ and reappeared at its final destination, where it remained visible for an additional $470 \mathrm{msec}$ before the participants were allowed to respond. This kept the timing analogous to the short interstimulus interval condition Lleras and Moore (2003) used to elicit apparent motion, with the target-plusmask presentation rate $(30 \mathrm{msec})$ and time between target-plus-mask presentation and potential responses $(500 \mathrm{msec})$ equaling those of Experiment 2. This produced apparent motion that was much more rapid than that in Experiment 2 because the mask took $30 \mathrm{msec}$ rather than $500 \mathrm{msec}$ to travel to its final destination.

\section{Results and Discussion}

The percentages of correct target responses are depicted in the right portion of Figure 5 (see the fast-interrupted motion conditions). A 2 (masking: masked vs. unmasked) $\times$ 2 (direction of motion: toward vs. away) repeated measures ANOVA revealed main effects of masking $[F(1,8)=$ $50.94, p<.001]$ and mask direction $[F(1,8)=11.42, p<$ $.01]$. Importantly, these effects were qualified by an in- teraction between masking and mask direction $[F(1,8)=$ 25.63, $p<.001]$ that was analogous to that observed in Experiment 2. With a Fisher's LSD of 7.5\%, masking was significant when the dot mask moved toward the target locations (masking effect $=22 \%$ ) but not when it moved outward (masking effect $=-5 \%$, with masking being nonsignificantly reversed). When the mask moved toward the target, the masking effect was larger here $(22 \%)$ than in Experiment $2(9 \%)[F(1,27)=11.64, p<.01]$. Because the masking object moved over 16 times faster here, traversing the distance in $30 \mathrm{msec}$ rather than $500 \mathrm{msec}$, the same amount of attention may not have been allocated to the target before the mask interfered with perception.

It is possible that we failed to find masking in the away condition because our displays, like those used in Experiment 2, did not contain distracting items; in Lleras and Moore's (2003) experiments, seven distractors appeared with the target. Di Lollo et al. (2000) documented that object-substitution masking increases with the number of distractors. According to Di Lollo et al., variables that impair target identification will increase masking; as the number of distractors increases, the total time for attention to make contact with the target increases $\left(t_{c}\right.$ in their computational model of object substitution, CMOS) and masking increases. Because we did not have distractors in Experiments 2 and 3, single-object morphing may have gone undetected. However, distractors were not necessary to detect masking when the mask moved toward the location where the target had been. By presenting distracting items that appear and offset at the same time as the target in Experiment 4 , we hoped to uncover masking in the away motion condition. In Experiment 4, we presented the distracting letter $\mathrm{H}$ in the quadrants that had previously been unoccupied, and the briefly masked control method was used (as in Experiment 1). It was predicted that adding distracting 
items would allow us to replicate the single-object morphing effect reported by Lleras and Moore when the mask moves away from the target location. In addition, masking should still arise from two-object interference when the mask moves toward the target location.

\section{EXPERIMENT 4}

In Experiment 4, we used the briefly masked control method. Here, object-substitution masking was characterized by decreased accuracy with increases in mask duration. By using the briefly masked control method, we were able to assess the generality of our results. By using distracting items (as Lleras \& Moore, 2003, had done) we predicted masking in both the away and toward conditions.

\section{Method \\ Participants. Fifteen students from Bates College participated for extra credit in an introductory psychology course. All reported having normal or corrected-to-normal vision. \\ Materials and Procedure. The materials and procedure were identical to those used in Experiment 3 with the following excep- tions. Here, the mask always appeared in the same quadrant as the target (i.e., the target was always masked) and the distracting letter $\mathrm{H}$ appeared in each of the three previously unoccupied locations (the location of the target and distracting letters was chosen randomly from trial to trial). As in Experiment 3, the mask was presented along with the target for $30 \mathrm{msec}$, disappeared for $30 \mathrm{msec}$, and then reappeared at its final destination. Unlike in Experiment 3, however, the mask remained visible for 16,250 , or $1,000 \mathrm{msec}$ before the participants were allowed to respond. The direction of the mask's movement (toward or away from the target location) and the identity of the target (E or F) again varied randomly across trials.}

\section{Results and Discussion}

The percentages of correct target responses are depicted in Figure 6. A 3 (mask duration: 16, 250, or 1,000 msec) $\times$ 2 (direction of motion: toward vs. away) repeated measures ANOVA yielded a main effect of mask duration $[F(2,28)=8.45, p<.001]$. Importantly, this effect was qualified by an interaction between the effects of mask duration and mask direction $[F(2,28)=3.89, p<.05]$. With a Fisher's LSD of 4\%, object-substitution masking was significant when the mask moved toward the target location (masking effect $=11 \%$ ) and when it moved away from the target location (masking effect $=5 \%$ ). These data replicate (1) the masking effects reported by Lleras and Moore (2003) when the mask moved away from the location where the target had been and (2) the masking effects reported in Experiments 2 and 3 when the mask moved toward the location where the target had been.

\section{GENERAL DISCUSSION}

Object-substitution masking likely reflects processing at both the image level and the object level (Breitmeyer \& Ogmen, 2000; Di Lollo et al., 2000; Lleras \& Moore, 2003). When considered in conjunction with the results of Lleras and Moore, we believe the present results indicate that two different mechanisms produce object-level masking: single-object morphing (as described by Lleras

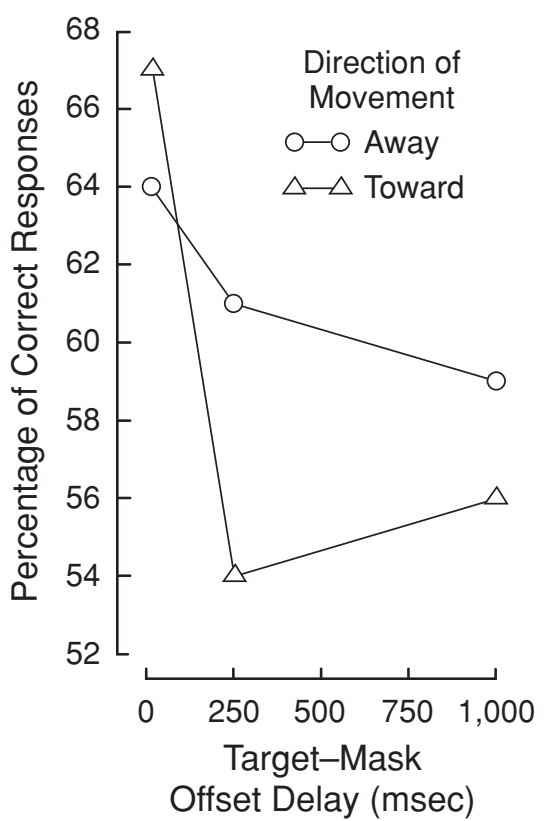

Figure 6. Experiment 4 accuracy rates as a function of mask duration and direction of movement.

\& Moore, 2003) and two-object interference. The contribution each of these makes to object-level masking likely varies with the experimental conditions. In Experiments $2-4$, two-object interference made a larger contribution to object-level masking than did single-object morphing, yet we acknowledge that, under different experimental conditions, single-object morphing might make a larger contribution.

\section{Masking in 3-D Space}

In Experiment 1, masking was obtained when the mask and the target appeared in the same depth plane and when the mask was seen in front of the target; masking was eliminated when the target was seen in front of the mask. Though it is clear that depth manipulations successfully influenced object-substitution masking in the manner predicted by two-object interference, it remains possible that the results from Experiment 1 were actually a consequence of single-object morphing, if one assumes that (1) looming motion was more easily seen (or more strongly seen) than was receding motion and (2) motion was seen across depth planes even though the conditions were designed to reduce the likelihood of this occurring. That is, in Experiment 1 , no slanting surface was presented on which the objects would more easily appear to move in depth, and the binocular disparity was large. Although He and Nakayama (1994) found that movement can, and will, occur across depth planes, especially when a surface (or an illusory surface) is provided on which the objects can appear to move, under conditions similar to those of Experiment 1, there is a preference to see motion within the same depth plane rather than across depth planes. However, unlike He and Nakayama, we did not use ambiguous motion displays; 
perhaps the lack of a competing motion signal encouraged our participants to see motion across depth planes. Despite this, it is unclear whether single-object morphing is influenced by the type of behavioral urgency that looming objects may produce (Franconeri \& Simons, 2003), and, more importantly, perceived motion across depth planes cannot explain the data from Experiments 2, 3, and 4. In Experiments 2-4, the mask did not move in depth and, as such, did not appear to be either looming or receding.

\section{Attention to the Mask}

Neill et al. (2002) demonstrated that masking effects increase as attention to the mask increases (or as attention to the target decreases). In Experiment 2, the mask remained visible and moved across the screen, perhaps attracting attention. In Experiments 3 and 4, the mask did not remain on the screen as it moved, but the movement of the mask may have attracted attention. Hence, one potential concern is that the masking effects reported in Experiments 2-4 might solely reflect this misallocation of attention, rather than object-substitution masking, per se. However, this possibility is easily ruled out, because the mask also moved in the unmasked conditions of Experiments 2 and 3 (and in Experiment 2, the mask remained visible in the unmasked conditions). Similarly, in Experiment 4, the mask moved in both the immediate-offset and the delayed-offset conditions. Consequently, the masking effects reported in Experiments 2 and 3 (i.e., the difference in accuracy in the unmasked and the masked conditions) and Experiment 4 (i.e., the difference in accuracy in the immediate- and the delayed-offset conditions) cannot simply reflect a form of attentional capture by the moving masks. Additionally, since the mask moved in both the away and the toward condition, if the mask did capture attention, this should have occurred equally in both the away and the toward condition, yet masking in Experiments 2-4 was consistently larger when the mask moved toward the target.

Similarly, the location of the mask (and other distractors) relative to the location of the target might influence attention and performance. Masks or distractors distant from the target's location might promote more divided attention, thereby reducing target accuracy. In fact, accuracy was lower when the mask started slightly adjacent to (towardmotion-masked condition), rather than surrounding (awaymotion-masked condition), the target. However, accuracy was much higher in the unmasked conditions relative to that in the masked conditions of Experiments 2 and 3, in which attention was clearly the most divided. Similarly, in Experiment 4, distracting items were presented simultaneously with the target, helping to ensure that attention was initially divided in all conditions; yet performance in the immediate-offset conditions was higher than performance in the delayed-offset conditions. As such, the data from Experiments 2-4 do not simply reflect the degree to which attention was initially divided across the display.

\section{Components of Object-Substitution Masking}

When displays use motion that originates at the target location and moves away, object-substitution masking is partly mediated by the updating of a single object token, as in Lleras and Moore's (2003) experiments and in the present Experiment 4 (away-motion condition). However, masking is also affected by two-object interference when the mask is seen moving toward the target, as demonstrated in the present Experiments 2, 3, and 4. In fact, under the conditions reported herein, two-object interference made a larger contribution to object-level masking than did single-object morphing; nevertheless, under different conditions, single-object morphing might make a larger contribution to object-level masking. When 3-D static displays are used and the masking dots surround the target, as in Experiment 1, masking occurs when the mask and the target are likely seen as separate object tokens (because they are perceived at different depths, and no slanting background surface is present to induce motion across depth; cf. He \& Nakayama, 1994). Thus, the singleobject morphing account cannot, unaltered, explain all of the data reported herein. Nor can these data be fully explained by image-level effects, because image-level variables (mask complexity, distance between target and mask contours, etc.) were identical in Experiment 1, yet masking was significantly less when the mask was perceived behind the target relative to when the mask was perceived in front of the target. Likewise, in Experiments 2, 3 , and 4, image-level variables (e.g., distance between the target and the mask contours) were likely larger in conditions that yielded less masking (the away-motion condition; see Note 2). Instead, these data indicate that both twoobject interference and single-object morphing contribute to object-level masking.

\section{Mechanism That Produces Two-Object Interference}

We envision object files as containing location information that is continually being updated, and we imagine that this might occur in a manner analogous to that proposed by Moore and Enns (2004). If location information were not stored in the object file, masking would not be influenced by the relative locations of the target and the mask. According to Moore and Enns, object updating (which includes updating location and form information in an object file) is the mechanism responsible for objectsubstitution masking, as well as a perceptual illusion referred to as the flash-lag effect (an illusion in which a flashed object erroneously appears to lag behind a continuously moving object, when the two are in actuality physically aligned). In the present experiments, if the target and the mask ended in competing locations, either because the mask was located in the same depth plane or in front of the target or because the mask stopped moving at the location where the target had been, we propose that the object representations would interfere with one another, thereby reducing target accuracy. However, it remains unclear whether the masking object's representation supplants the target following cyclical updates or whether the interference between these object files occurs at retrieval (rather than in the maintenance of the target's object file). We note that, although our interpretation of object updat- 
ing is similar to Moore and Enns's account-in the sense that location information is said to be stored and updated at the object level - it also differs from their account in one significant way. Moore and Enns hypothesize that objectsubstitution masking reflects the updating of a single object file (one-object morphing), whereas we hypothesize that object-substitution masking also reflects interference between two spatially competing object files (two-object interference).

\section{Conclusion}

By examining masking with 3-D (Experiment 1) and with apparent motion (Experiments 2-4) displays, we believe we have provided compelling evidence that objectlevel masking arises from both two-object interference and single-object morphing.

\section{REFERENCES}

BReITMEyer, B. G. (1984). Visual masking: An integrative approach. New York: Oxford University Press.

Breitmeyer, B. G., \& Ogmen, H. (2000). Recent models and findings in visual backward masking: A comparison, review, and update. Perception \& Psychophysics, 62, 1572-1595.

Di Lollo, V., Enns, J. T., \& Rensink, R. A. (2000). Competition for consciousness among visual events: The psychophysics of reentrant visual processes. Journal of Experimental Psychology: General, 129, 481-507.

EnNs, J. T., \& Di Lollo, V. (1997). Object substitution: A new form of masking in unattended visual locations. Psychological Science, 8, 135-139.

EnNs, J. T., \& Di Lollo, V. (2000). What's new in visual masking? Trends in Cognitive Sciences, 4, 345-352.

Franconeri, S. L., \& Simons, D. J. (2003). Moving and looming stimuli capture attention. Perception \& Psychophysics, 65, 999-1010.

He, Z. J., \& NaKayama, K. (1994). Apparent motion determined by surface layout not by disparity of three-dimensional distance. Nature, 367, 173-175.

Kahan, T. A., \& Mathis, K. M. (2002). Gestalt grouping and common onset masking. Perception \& Psychophysics, 64, 1248-1259.

Kahneman, D., Treisman, A., \& GibBs, B. J. (1992). The reviewing of object files: Object-specific integration of information. Cognitive Psychology, 24, 175-219.

Kraut, R., Olson, J., Banaji, M., Bruckman, A., Cohen, J., \& Couper, M. (2004). Psychological research online: Report of the board of scientific affairs' advisory group on the conduct of research on the Internet. American Psychologist, 59, 105-117.
Lleras, A., \& Moore, C. M. (2003). When the target becomes the mask: Using apparent motion to isolate the object-level component of object substitution masking. Journal of Experimental Psychology: Human Perception \& Performance, 29, 106-120.

McGraw, K. O., Tew, M. D., \& Williams, J. E. (2000). The integrity of Web-delivered experiments: Can you trust the data? Psychological Science, 11, 502-506.

Moore, C. M., \& EnNs, J. T. (2004). Object updating and the flash-lag effect. Psychological Science, 15, 866-871.

Moore, C. M., \& Lleras, A. (2005). On the role of object representations in substitution masking. Journal of Experimental Psychology: Human Perception \& Performance, 31, 1170-1180.

Neill, W. T., Hutchison, K. A., \& Graves, D. F. (2002). Masking by object substitution: Dissociation of masking and cuing effects. Journal of Experimental Psychology: Human Perception \& Performance, 28, 682-694.

Schneider, W., Eschman, A., \& Zuccolotto, A. (2002a). E-Prime reference guide. Pittsburgh: Psychology Software Tools.

Schneider, W., Eschman, A., \& Zuccolotto, A. (2002b). E-Prime user's guide. Pittsburgh: Psychology Software Tools.

\section{NOTES}

1. Moore and Lleras (2005) used the phrase object-mediated updating to describe the updating of a single object file, but we have chosen to use the nomenclature single-object morphing to highlight the difference between this account and the two-object interference account of objectlevel masking.

2. The masking contours are initially closer to the target's contours when the mask starts in the same location and moves away, relative to when the mask starts in a separate location and moves toward the location where the target had been. This would likely result in more image-level masking when the mask initially surrounds the target (the away-motion condition). However, the mask is presented for a longer duration at the target location when the mask starts in a distant location, relative to when the mask initially surrounds the target, possibly contributing to larger image-level masking when the mask originates in a distant location (the toward-motion condition). But this possibility is unlikely, because it took $500 \mathrm{msec}$ for the mask to reach its final position in Experiment 2, well after the target had been presented and removed from the screen. Therefore, it seems likely that image-level masking will be maximal when the mask initially surrounds the target (the away-motion condition). (Note that it took only $30 \mathrm{msec}$ for the target to reach its final position in Experiments 3 and 4, making it somewhat less clear which condition-toward or away_would produce greater image-level masking.)

(Manuscript received February 25, 2005; revision accepted for publication June 8, 2005.) 